\title{
GC-ICP-MS/MS Instrumental Set-up for Total and Speciation Sul- fur Analysis in Gasolines using Generic Standards
}

\author{
Laura Freije-Carrelo, Javier García-Bellido, Francisco Calderón-Celis, Mariella Moldovan*, Jorge Ruiz \\ Encinar*
}

Department of Physical and Analytical Chemistry, University of Oviedo, Julián Clavería 8, 33006, Oviedo, Spain

\begin{abstract}
Quantitative characterization of sulfur-containing petroleum derivatives is mainly limited by the large number of potential targets present and the matrix effects suffered due to the high carbon-containing matrices. Herein we describe the instrumental modifications required in a commercial GC-ICP-MS/MS instrument, and their corresponding optimization, aiming to turn it into a compound-independent quantitative technique for both total and speciation sulfur analysis in gasolines. Additionally, carbonderived matrix effects were made negligible for direct and fast total $\mathrm{S}$ analysis, making the use of relatively complex isotope dilution strategies not necessary anymore. Absolute detection limit of $0.3 \mathrm{pg} \mathrm{S}$ was achieved, which is, to the best of our knowledge, more than one magnitude order below the ones reported for other sulfur GC selective detectors. Precision was below 3\% RSD. Total analysis was performed by flow injection analysis through a transfer line and external calibration, whereas speciation analysis was carried out by chromatographic separation and internal standardization. In both cases, simple generic standards were used, which enabled to get rid of specific S-containing standards (sometimes not available or/and unstable). The proposed method was successfully applied to total and speciation sulfur analysis of a commercial gasoline sample and validated with a certified reference material (ERM-EF213) gasoline. The approach has proved to be simple, fast, robust and convenient for implementation in routine laboratories as demonstrated by the successive analysis of 50 gasoline samples in 3 hours without any instrumental drift.
\end{abstract}

Petroleum derivatives are highly complex samples consisting of a mixture of hydrocarbons and heteroatom-containing compounds, the most abundant one being sulfur (up to a $8 \%$ in crude oil), which can be found in different functional groups, mainly thiols, sulfides and thiophenes. The relevance of these compounds lies in their corrosive nature, that causes catalyst poisoning in refining processes and in their pollution impact on the environment. ${ }^{1,2}$ Maximum permissible sulfur contents are already regulated. For instance, a legal limit of $10 \mathrm{ppm}$ (w/w) of sulfur in diesel fuels and gas oils has been established in Europe (Directive 2009/30/EC). ${ }^{3}$ In addition to total sulfur analysis, quantitative speciation of the individual $\mathrm{S}$ containing compounds is also advisable in petroleum samples, aiming at the optimization of the desulfuration processes and/or in environmental studies. ${ }^{1,4}$

Different techniques for direct total sulfur analysis in petroleum derivatives have been reported, such as wavelengthdispersive X-ray fluorescence ${ }^{5}$ or ultraviolet fluorescence. ${ }^{6}$ These techniques, nonetheless, suffer from poor limits of detection (LODs), around $1 \mathrm{ppm}$, poor precision and accuracy and in most cases matrix effects have to be accounted for. On the other hand, speciation analysis of the S-containing compounds in light and middle distillates is commonly performed by gas chromatography (GC) coupled to sulfur selective detectors. Importantly, quantification of these sorts of samples might be challenging given the high number of S-containing compounds present. Therefore, compound-independent calibration (CIC) would be very convenient, thus avoiding the use individual standards (sometimes not available or/and unstable) for each target compound. Flame photometric detector (FPD) and pulsed flame photometric detector (PFPD) have been used. However, responses in FPD are usually non-linear, relatively non-uniform and can be quenched by co-elution of hydrocarbons. ${ }^{7,8}$ Atomic emission detector (AED) can be also applied for $\mathrm{S}$ specific detection but there is considerable controversy with regard to the possibility of performing CIC. ${ }^{9,10}$ Sulfur chemilumiscent detector (SCD) proved to be superior to other detectors, being widely used for sulfur speciation in petroleum derivatives with LODs in the low ppb range. However, heavy organic matrix can lead to significant signal drift, resulting in stringent operation and maintenance procedures. ${ }^{9}$

Alternatively, inductively coupled plasma mass spectrometry (ICP-MS) could be a really sensitive and convenient technique for both total and speciation sulfur analysis able to provide species-independent response. Nevertheless, direct liquid total analysis of petroleum samples by ICP-MS requires conscientious selection of the introduction technique to prevent large quantities of carbon arriving to the plasma, either by low-flow nebulizers, cooled spray chambers or alternative procedures. ${ }^{11-13}$ Direct injection by thermal vaporization and ICP-MS was also employed. Unfortunately, matrix effects and signal-drifts caused by co-elution of hydrocarbons demanded for the application of isotope dilution using ${ }^{34} \mathrm{~S}$-labelled volatile standards. Even so, only $0.1 \mu \mathrm{L}$ of sample could be injected, and nitrogen gas bracketing injections were needed. ${ }^{14}$ On the other hand, ICP-MS can also be used as GC selective detector for sulfur speciation in petroleum-related samples. ${ }^{15}$ Once more, the main difficulty lies on the carbon matrix effects caused by co-elution of hydrocarbons with the Scontaining compounds so accurate sulfur speciation could only be performed by species-specific ${ }^{16}$ or online ${ }^{17}$ isotope dilution. Although this last approach avoids the use of individual stand- 
ards, it still requires the synthesis, characterization and online addition of a ${ }^{34} \mathrm{~S}$-labelled gas standard that is more complicated than in the case of the use of ${ }^{34} \mathrm{~S}$-labelled standards in HPLC. Recently, the development of ICP-MS/MS technology opened the door to interference-free sulfur detection, as already demonstrated in numerous applications. ${ }^{18-20}$ Although sulfur profiles have been already reported using GC-ICP$\mathrm{MS} / \mathrm{MS},{ }^{21}$ the application of ICP-MS/MS as quantitative compound-independent sulfur GC detector has not been carefully optimized and demonstrated so far. This work presents a new instrumental GC-ICP-MS/MS set-up able to provide both total and speciation sulfur quantitative analysis at the very low ppb level in petroleum derivatives without the need for complex isotope dilution procedures.

\section{EXPERIMENTAL SECTION}

\section{Standards and samples}

Dibutylsulfide (DBS, purity 100\%), ethyl phenyl sulfide (EPS, 97\%), benzothiophene (BT, 97\%), methylbenzothiophene (MBT, 96\%), dibenzothiophene (DBT, 98\%), bromocyclohexane $(\mathrm{CXB}, 99 \%)$ and hexane were purchased from Sigma-Aldrich. Dimethyl sulfide (DMS) in Argon gas standard $(2.9 \pm 0.3 \mathrm{ppm} \mathrm{S}, \mathrm{v} / \mathrm{v})$ was obtained from Linde AG (Munich, Germany). $\mathrm{CO}_{2}$ : $\mathrm{Ar}(10: 90)$ and $\mathrm{O}_{2}: \mathrm{Ar}$ (20:80) were supplied by Air Liquide (Madrid, Spain). Commercial gasoline was obtained from a regular gas station (Asturias, Spain). Certified reference material ERM-EF213, consisting of a real "sulfur-free" gasoline $(9.1 \pm 0.8 \mathrm{ppm} \mathrm{S}$, w/w) was obtained from BAM (Berlin, Germany).

\section{Instrumentation}

A GC 7890B (Agilent Technologies, CA, USA) was coupled to a Triple Quad ICP-MS, ICP-MS/MS (Agilent 8800, Tokyo, Japan) by means of a heated GC-ICP-MS interface (Agilent Technologies). A gas sampling valve (GSV) located outside the oven and heated at $200^{\circ} \mathrm{C}$, was connected to the GC inlet in order to inject $1 \mathrm{~mL}$ of the DMS gas standard when necessary. An Ar pipe was taken out of the oven and attached to the heated GSV box. GC and ICP-MS/MS conditions are detailed in Table S-1 of Supporting Information and a diagram of the set-up is shown in Figure 1. Total or speciation sulfur analysis were performed using an inert transfer line (fused silica deactivated, $5 \mathrm{~m} \mathrm{x} 0.32 \mathrm{~mm}$ i.d., Agilent Technologies) or a J\&W HP-5 column (30 m x $0.32 \mathrm{~mm}$ i.d. x 0.25 $\mu \mathrm{m})$, respectively. Manual injection was used for liquid samples. $\mathrm{CO}_{2}$ :Ar (10:90) was introduced online using a Tconnection and a Mass Flow Controller (MFC) PR4000B (MKS Instruments, Andover, USA).

\section{Procedures}

\section{Assessment of the species-independent sulfur response}

$1 \mathrm{~mL}$ of DMS was injected by the GSV and the transfer line at different temperatures ( 80 to $320^{\circ} \mathrm{C}$ ). Sulfur areas were computed both with the pre-heating Ar pipe inside and outside the oven. Then, $1 \mu \mathrm{L}$ of a mixture $(0.3 \mathrm{ppm} \mathrm{S}$, w/w $)$ of DBS, EPS, BT, MBT and DBT was injected through a GC column, both using splitless and split modes (1:6 and 1:10 ratios). Response factors and recoveries were computed using DBS as internal standard (IS).

\section{Assessment of carbon matrix effects for sulfur detection}

$1 \mathrm{~mL}$ of DMS was injected through the GSV and the transfer line at increasing flows of the $\mathrm{CO}_{2}$ :Ar mixture (from 2 to 5
$\mathrm{mL} / \mathrm{min}$ in steps of $0.5 \mathrm{~mL} / \mathrm{min}$ ). To do so, $\mathrm{CO}_{2}:$ Ar was mixed online with the option $\mathrm{O}_{2}$ :Ar gas $(8 \%)$ and dilution/option $\mathrm{Ar}$ gas through a T-connection 5/32" (John Guest, Middlesex, $\mathrm{UK}$ ), as shown in Figure 1. $\mathrm{O}_{2}$ :Ar gas was added to prevent carbon build up on the cones during the working time.

\section{Direct total volatile sulfur analysis by GC-ICP-MS/MS}

Total sulfur analysis was carried out by injecting the sample into the transfer line. Detailed conditions are shown in Table $\mathrm{S}-1$. External calibration curve was built injecting $1 \mu \mathrm{L}$ of a mixture of BT in hexane (3-12 ppm S, w/w) and CXB, added as IS (c.a. $13 \mathrm{ppm} \mathrm{Br}$ ). Then, $1 \mathrm{~g}$ of gasoline was spiked as well with $13 \mathrm{ppm} \mathrm{Br}$. For standard additions, $0.2 \mathrm{~g}$ of a mixture containing increasing concentration of BT standard and constant concentrations of $\mathrm{CXB}$ was added to $10 \mathrm{~g}$ of the commercial gasoline.

\section{Sulfur speciation analysis by GC-ICP-MS/MS}

Detailed conditions are shown in Table S-1. Commercial and CRM gasoline samples were spiked with DBT as IS (0.5 and $2 \mathrm{ppm}$ S, respectively).

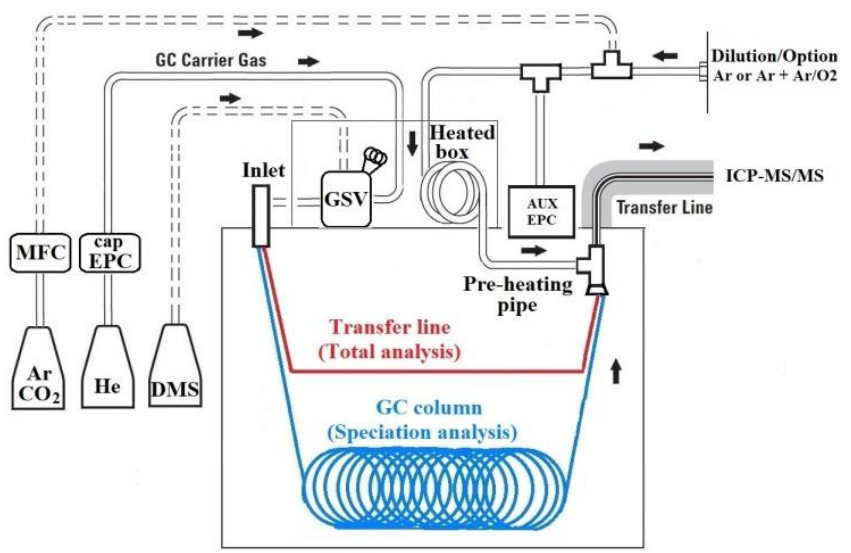

Figure 1. Diagram of the GC-ICP-MS/MS set-up for total analysis (transfer line, red line) or speciation analysis (GC column, blue line). Dimethyl sulfide gas standard (DMS) and Ar: $\mathrm{CO}_{2}$ (dotted lines) were only used for the assessment of species-independent response and/or matrix effects.

\section{RESULTS AND DISCUSSION}

\section{Instrumental modifications to obtain species- independent sulfur response}

When performing CIC in GC-ICP-MS, it is required the quantitative transmission of all the compounds along the GC and the GC-ICP-MS interface, as well as species-independent ionization in the ICP-MS plasma. In the new commercial Agilent GC-ICP-MS interface, the flow from the column is mixed online with the Ar carrier (mix of the "dilution" and "option" flows) previously heated by means of a pre-heating pipe (ca. $4.5 \mathrm{~m}$ ) that is located inside the GC oven. Therefore, the temperature of the Ar carrier changes dramatically along a regular GC gradient. To evaluate whether the $\mathrm{S}$ response could be influenced by this effect triplicate injections of DMS gas were made using the GSV and the fused silica transfer line, at different oven temperatures $\left(80-320^{\circ} \mathrm{C}\right)$. In this way, inlet and column issues were avoided and only the oven temperature played a role. Surprisingly, as illustrated in Figure 2, DMS S areas increased significantly during the experiment (up to a $55 \%$ ), hampering therefore sulfur CIC. Such results pointed to the likelihood that Ar carrier flow, which influences a lot on the sensitivity, could change significantly as the temperature 
of the oven increases. In order to demonstrate this assumption an auxiliary electronic pressure control (Aux EPC) was installed in the GC and directly connected to Ar flow line using a "T" (Figure 1) to monitor the back-pressure along the gradient. As can be seen in Figure S-1, back-pressure increased significantly $(56 \%)$ with temperature of the oven. We decided then to take the pipe outside the oven and place it in the GSV box in order to still heat the Ar carrier but at a constant temperature of $200^{\circ} \mathrm{C}$. Pressure values measured under such instrumental conditions remained unchanged with temperature (Figure S-1, $<7 \%$ variation). Moreover, triplicate injections of DMS gas were then repeated and the $S$ areas obtained were stable with temperature (3\% RSD), as clearly shown in Figure 2.

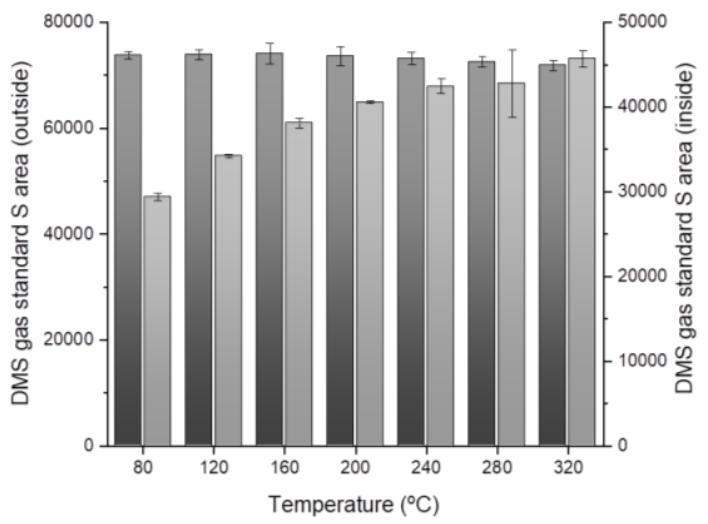

Figure 2. Peak areas for the DMS gas standard from 80 to $320^{\circ} \mathrm{C}$ with the Ar pre-heating pipe inside (light grey) and outside the oven (dark grey). Uncertainty bars correspond to 2 standard deviations $(\mathrm{n}=3)$.

Once the stability of the $\mathrm{S}$ response factor along the GC was achieved, a solution containing five $S$ compounds (DBS, EPS, BT, MBT and DBT), which represent the families typically present in petroleum derivatives, was injected onto a GC column (Figure S-2) using different split ratios (splitless, 1:6 and 1:10). The first eluting compound (DBS) was used as IS for the quantification of the other four so that possible variations in the $S$ response factor due to different transmission efficiencies at the inlet/column could be assessed. As shown in Table S-2, recoveries ranging from 97 to $104 \%$, were obtained, demonstrating that CIC of S can be safely carried out with the customized GC-ICP-MS/MS set-up.

LOD ranged from $380-420$ ppt $\mathrm{S}$ depending on the species (absolute detection limit of ca. $0.3 \mathrm{pg} \mathrm{S}$ ). To the best of our knowledge, this LOD is more than one magnitude order below the ones reported for other S selective detectors, such as SCD or ICP-MS (both in ppb levels) ${ }^{9}$, and similar to the those obtained GC-ICP-MS/MS instrument. ${ }^{21}$ Interestingly, LODs reported in this last case were more than one order of magnitude different depending on the S-containing species analyzed, what seems to support the need for the instrumental modifications proposed herein to obtain sulfur CIC when using the commercial GC-ICP-MS/MS instrument. Linear range obtained covers more than 2 orders of magnitude (1-360 ppb S, Figure S-3).

\section{Assessment of carbon matrix effects for sulfur detection in petroleum derivatives}

It is well known that co-elution with carbon-containing compounds may produce sulfur matrix effects, and strategies such as isotope dilution were developed to overcome them. ${ }^{15,17}$
Therefore, once species-independent S signal was demonstrated, we wanted to assess the occurrence of carbon matrix effects in the plasma. In order to keep full control on the amount of carbon arriving to the plasma, injections of the DMS were made through the GSV connected to the transfer line, while flows of the $\mathrm{CO}_{2}$ :Ar mixture (10:90) were added online, as shown in Figure 1. Carbon signal was monitored as ${ }^{29} \mathrm{CO}^{+}$to obtain a rough profile of the added carbon. A minimum flow of $2 \mathrm{~mL} / \mathrm{min}$ (limited by the MFC used) and a maximum flow of $5 \mathrm{~mL} / \mathrm{min}$ were tested, corresponding to 107 and $268 \mu \mathrm{g}$ $\mathrm{C} / \mathrm{min}$, respectively. Note that $268 \mu \mathrm{g} \mathrm{C} / \mathrm{min}$ is equivalent to a flow injection analysis (FIA) of $1 \mu \mathrm{L}$ of pure gasoline eluting in 2.5 min. FIAgram and DMS sulfur areas obtained are shown in Figure 3-A and 3-B, respectively. Remarkably, both the RSD of the S areas for individual triplicates at each carbon content and the RSD for all injections were around $1 \%(n=24)$, proving that occurrence of matrix effects was negligible within the tested range.
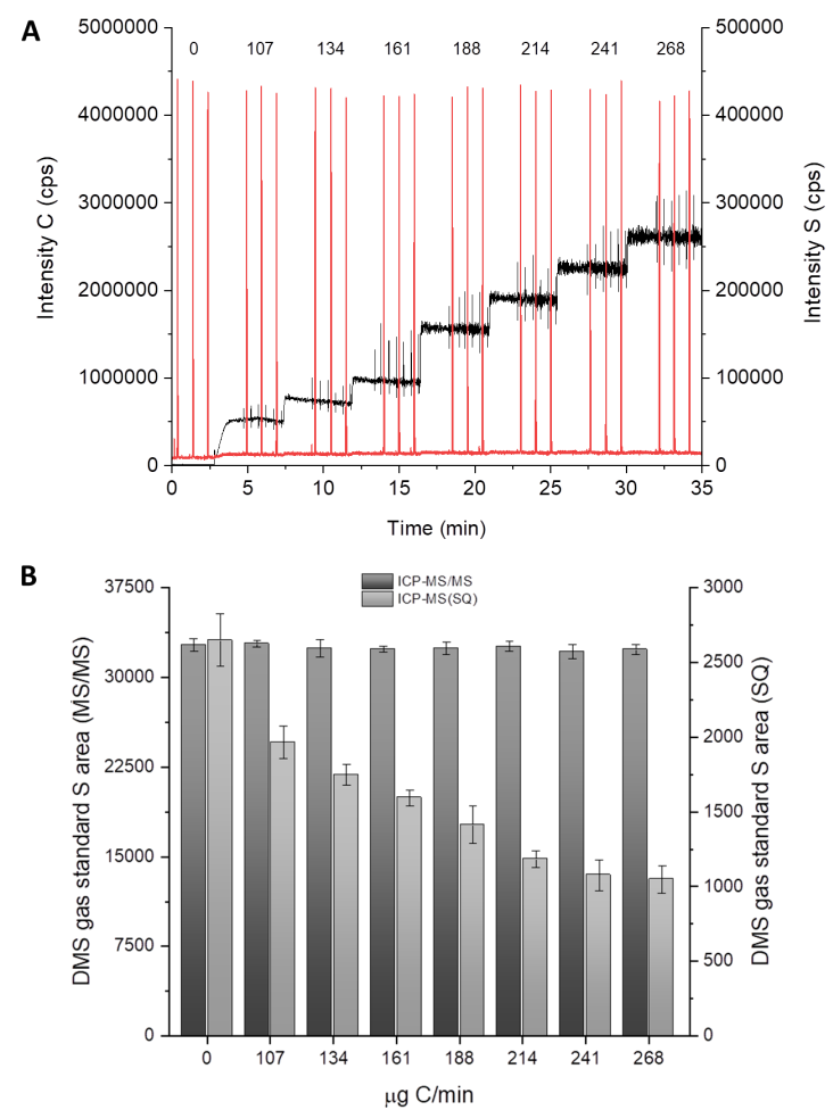

Figure 3. Matrix effects evaluation from 0 to $268 \mu \mathrm{g} \mathrm{C} / \mathrm{min}$. A) Carbon (black) and sulfur (red) FIAgrams obtained by GC-ICPMS/MS for triplicate injections of DMS gas standard. B) DMS areas computed by GC-ICP-MS/MS and GC-ICP-MS (SQ). Uncertainty bars correspond to 2 standard deviation $(n=3)$.

These results nonetheless are in disagreement with previous studies in which analysis of S-containing species in petroleum products showed signal variations attributable to carbon matrix effects. ${ }^{17}$ In order to clarify this issue, a study under conditions that bore a close resemblance to such works (RF power $700 \mathrm{~W}$, no cell gas, no optional $\mathrm{Ar}: \mathrm{O}_{2}$ gas, single quad, SQ) was carried out. ${ }^{17}$ FIAgram and computed areas by GC-ICPMS are shown in Figure S-4 and Figure 3-B, respectively. In contrast to our results, this second set showed a significant 
correlation between $\mathrm{S}$ signal and carbon added to the plasma. Interestingly, this decrease is consistent with the negative peaks observed in the $\mathrm{S}$ background and caused by hydrocarbon co-elution when using GC-ICP-MS (SQ). ${ }^{17}$ Finally, we wanted to corroborate that matrix effects were also negligible when analyzing a C-containing matrix in the liquid phase. For this purpose, direct injection in splitless mode of $1 \mu \mathrm{L}$ of pure hexane was made while a sulfur continuous signal was monitored coming from the DMS gas mixed online with the $\mathrm{Ar}$ flow. As can be clearly seen in Figure S-5, elution of $1 \mu \mathrm{L}$ of hexane $(547 \mu \mathrm{g} \mathrm{C})$ during $2.5 \mathrm{~min}$ did not produce any distortion on the $\mathrm{S}$ continuous signal. Therefore, the use of the GCICP-MS/MS set-up proposed herein seems to allow S measurement without significant matrix effects what opens the door to sulfur CIC in high carbon-content samples without the need of isotope dilution.

\section{Direct total volatile sulfur analysis of gasoline by GC- ICP-MS/MS}

After assuring compound independent S signal and lack of carbon matrix effects, total analysis of commercial gasoline could be then carried out just by connecting a transfer line to the GC inlet. An external calibration curve was built using flow injections (FIA) of BT as external S-generic standard diluted in hexane. Split 1:10 was selected as demonstrated before it did not lead to any differential transmission for $\mathrm{S}$ containing compounds (see Table S-2). In order to compensate for likely differences in the volume injected (manual injection was used) CXB was selected as IS and added to both external standards and gasoline samples. Since FIA was used, IS selected must contain another ICP-detectable element different than S. A bromine-containing compound was selected as bromine species are not expected in gasolines and ICP-MS background for bromine is typically low. Moreover, CBX standard is stable and commercially available. Therefore, $\mathrm{S} / \mathrm{Br}$ peak area ratio was computed as analytical parameter. In parallel, a standard addition curve was also prepared by spiking increasing concentrations of BT to the commercial gasoline under study adding CXB as IS as well. Calibration graphs obtained for external calibration and standard additions are shown in Figure S-6. Slopes for both external calibration $(\mathrm{y}=8.99 \mathrm{x}+0.24)$ and standard addition curves $(\mathrm{y}=8.57 \mathrm{x}+4.15)$ were similar, confirming further the absence of matrix effects under the conditions used. Quantification by external calibration was preferred because of its simplicity. Total sulfur concentration obtained by external calibration for the commercial gasoline was found to be $6.1 \pm 0.3 \mathrm{ppm} S$, below the $S$ legal limit (10 ppm S). FIAgram for total sulfur analysis by external calibration and quantification results are shown in Figure 4.A and Table 1, respectively. CRM ERM-EF213, consisting of a real "sulfur-free" gasoline with certified total $S$ content $(9.1 \pm 0.8 \mathrm{ppm} S)$, was also analysed for validation purposes. As can be seen in Table 1, experimental result obtained using the proposed set-up $(8.8 \pm 0.4 \mathrm{ppm} \mathrm{S})$ was in excellent agreement with the certified value. Precision obtained was below $3 \%$ RSD for both real samples.

Table 1. Quantitative results for total and speciation sulfur analysis of CRM ERM-EF213 and commercial gasolines.

$$
\text { CRM Commercial }
$$

ERM-EF213 gasoline

Certified total S content $\quad 9.1 \pm 0.8 \quad-$

\begin{tabular}{lcc}
\hline Total analysis by FIA & $8.8 \pm 0.4$ & $6.1 \pm 0.3$ \\
\hline Speciation analysis & & \\
$\quad$ Thiophene & $6.58 \pm 0.39$ & $1.31 \pm 0.07$ \\
$\quad$ Monomethylthiophenes & $1.19 \pm 0.10$ & $0.19 \pm 0.04$ \\
$\quad$ Total S content & $9.3 \pm 0.6$ & $6.2 \pm 0.3$ \\
\hline
\end{tabular}

* All concentrations are given in ppm (w/w). Propagated uncertainties (95\% confidence level) are given $(n=3)$.

Finally, the feasibility of the proposed approach for routine analysis of total $\mathrm{S}$ content in petroleum derivatives was assessed. Successive injections of the CRM gasoline were carried out. Sample preparation was reduced to a simple spiking of CXB as IS and calibration was performed at the beginning of the experiment. A solution of BT in hexane (spiked with $\mathrm{CXB}$ ) was injected twice every 10 gasoline samples as QC standard. Results obtained are shown in Figure 4-B. As can be seen, no signal drift was apparent along the 60 injections performed ( 3 hours of total analysis time). Notably, RSD of the 50 gasoline samples was as low as $3.6 \%$. Similar precision $(3.0 \%, \mathrm{n}=10)$ was obtained for the QC standards. Interestingly, the long sequence of real complex samples did not produce any detrimental effect on the performance of the set-up. Moreover, absolute peak areas obtained for $\mathrm{S}$ and $\mathrm{Br}$ in the QC injections did not show any specific trend (7\% RSD), what seems to indicate that the sequence could have been extended without any problem. In fact, neither liner, transfer line nor ICP cones had to be cleaned after the analysis. Sample throughput was as high as 3 min per sample.
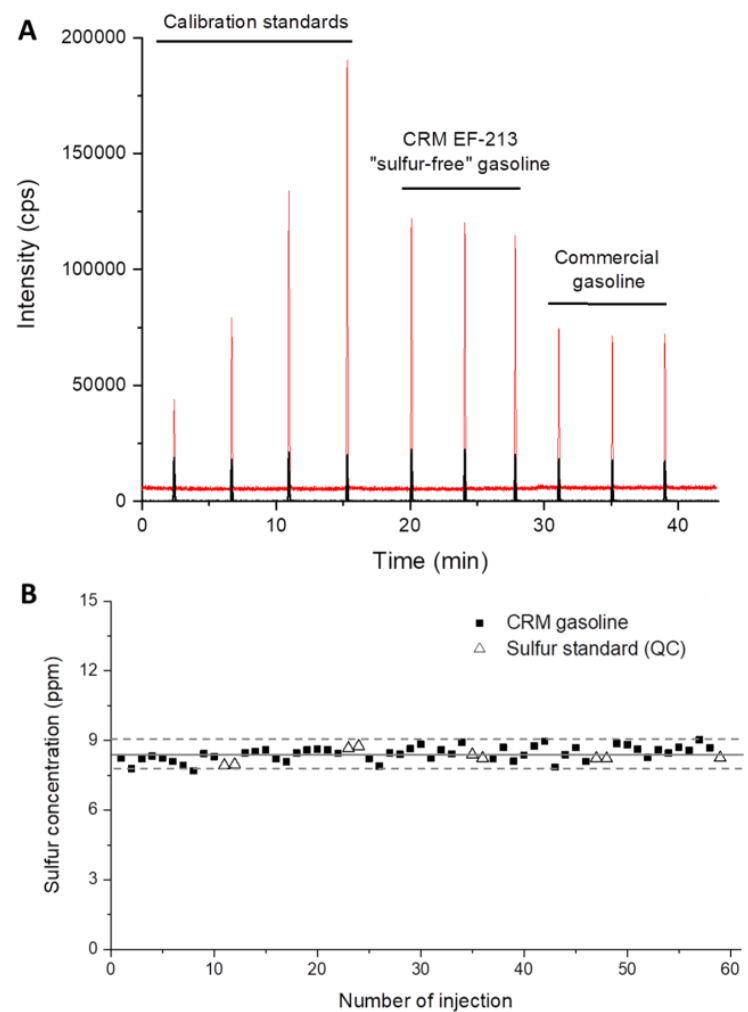

Figure 4. Total sulfur analysis of commercial and CRM gasolines by GC-ICP-MS/MS A) Sulfur (red) and bromine (black) FIAgram and B) Robustness study for 60 consecutive injections. Solid and dotted lines indicate the mean value and 95\% confidence interval, respectively. 


\section{Speciation sulfur analysis of gasoline by GC-ICP- MS/MS}

Speciation sulfur analysis was carried out for both the commercial and CRM gasolines by simple exchanging the transfer line by a GC column. DBT was added as IS as it is not naturally present in gasolines. Its computed $\mathrm{S}$ response factor was used for quantification of the S-species present. Chromatograms obtained are shown in Figures 5 and S-7 for the CRM and commercial gasolines. Quantitative results for the major species (thiophene and monomethylthiophenes isomers) as well as the total $\mathrm{S}$ concentration, calculated using the area sum of all the $\mathrm{S}$ peaks obtained in the chromatogram, are given in Table 1. Total sulfur contents by speciation analysis were found to be statistically indistinguishable to the direct total flow injection analyses, both for the commercial gasoline $(6.2 \pm 0.3 \mathrm{ppm} \mathrm{S})$ and CRM $(9.3 \pm 0.6 \mathrm{ppm} \mathrm{S})$, what internally validates our results and demonstrates again the negligible influence of the matrix in the direct analysis of total $\mathrm{S}$ content. Moreover, thiophene and monomethylthiophenes concentrations found in the CRM gasoline $(6.58 \pm 0.39$ and $1.31 \pm 0.07)$ fitted very well with the previously reported contents obtained using GC-ICP-MS and online isotope dilution analysis $\left(6.62 \pm 0.12\right.$ and $1.33 \pm 0.09$, respectively), ${ }^{17}$ which can be considered as reference method.

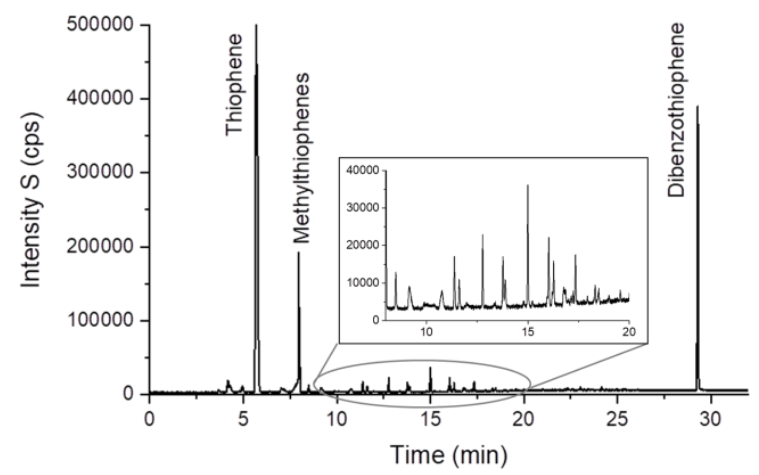

Figure 5. GC-ICP-MS/MS chromatogram of CRM EF-213 gasoline spiked with dibenzothiophene as internal standard.

\section{CONCLUSIONS}

We present herein the instrumental modifications and careful optimization required in a commercial GC-ICP-MS/MS instrument to make it possible to obtain S-species independent quantification. A number of independent experiments were also conducted in order to demonstrate the absence of C-based matrix effects when analyzing directly gasoline samples. Under such instrumental conditions isotope dilution strategies are not necessary anymore. These unique characteristics make the set-up developed a valuable alternative for total and speciation $\mathrm{S}$ analysis in petroleum-related samples using simple and generic S- and Br-standards with sulfur LODs as low as 400 ppt (w/w). Notably it is also simple, fast, robust and thus very convenient for its implementation in petroleum laboratories.

Total or speciation sulfur quantifications can be performed without any pre-treatment of the sample using one single instrumental set-up just by connecting the GC inlet either to a transfer line (total analysis) or to a column (speciation analysis). It is worth mentioning that the use of a GC equipped with two inlets, one connected to a transfer line and the other to the column, and both to the GC-ICP-MS transfer line by means of a two-hole ferrule, as previously described elsewhere for gas sample analysis, ${ }^{22}$ would simplify further the approach. Such integrated set-up could be useful for high-throughput total sulfur quantification (screening). Notably, robustness of the approach would allow long sequence analysis of such real samples while speciation could be later performed exclusively on those whose total S content would be significant.

Finally, most of the exceptional features obtained for $\mathrm{S}$ analysis could be extended to species containing other ICPdetectable elements and amenable to analysis by GC such as $\mathrm{P}-, \mathrm{Si}-, \mathrm{Br}-$ or Cl-containing compounds. ${ }^{23,24}$ This would extend the scope of application from petroleum derivatives to biogas, natural gas or environmental samples.

\section{ASSOCIATED CONTENT}

\section{Supporting Information}

Instrumental parameters, recoveries and chromatograms for a model mixture of sulfur compounds, figures regarding CIC and matrix assessment, calibration curves and chromatogram of the gasoline sample (PDF).

\section{AUTHOR INFORMATION Corresponding Authors}

*E-mail: moldovanmariella@uniovi.es, ruizjorge@uniovi.es

\section{ACKNOWLEDGMENT}

Spanish Ministry of Economy and Competitiveness is acknowledged for CTQ2016-79412-P project and F.C.C. grant (BES2014-068032). Principado de Asturias is acknowledged for IDI/2018/000166 project and L.F.C grant (BP16181).

\section{REFERENCES}

(1) Han, Y.; Zhang, Y.; Xu, C.; Hsu, C. S. Fuel. 2018, 221, 144-158.

(2) Yepez, O. Fuel 2005, 84, 97-104.

(3) Directive 2009/30/EC of the European parliament and of the Council of 23 April 2009 Off. J. Eur. Union. 2009, 140, 88-112.

(4) Andersson, J. T.; Hegazi, A. H.; Roberz, B. Anal. Bioanal. Chem. 2006, 386, 891-905.

(5) ISO 14596: 2009. Determination of sulfur content. Wavelengthdispersive X-ray fluorescence spectrometry.

(6) ISO 20846: 2011. Determination of Sulfur Content of Automotive Fuels-Ultraviolet Fluorescence Method.

(7) Del Río, D.; Rey, M.; Sedran, U.; De La Puente, G. Fuel Process. Technol. 2011, 92, 2278-2284.

(8) McKelvie, K. H.; Thurbide, K. B. Anal. Methods 2017, 9, 10971104

(9) Dettmer-Wilde, K.; Engewald, W; Practical Gas Chromatography. Springer-Verlag Berlin An, 2016.

(10) Chernetsova, E. S.; Revel'skii, a. I.; Revel'skii, I. a.; Zolotov, Y. A. J. Anal. Chem. 2010, 65, 770-784.

(11) Gutiérrez Sama, S.; Farenc, M.; Barrère-Mangote, C.; Lobinski, R.; Afonso, C.; Bouyssière, B.; Giusti, P. Energy Fuels 2018, 32, 4593-4605.

(12) Gajdosechova, Z.; Boskamp, M. S.; Lopez-Linares, F.; Feldmann, J., Krupp, E. M. Energy Fuels 2016, 30, 130-137

(13) Poirier, L.; Nelson, J.; Leong, D.; Berhane, L.; Hajdu, P.; LopezLinares, F.; Energy Fuels 2018, 30, 3783-3790.

(14) Heilmann, J.; Heumann, K. G. Anal. Bioanal. Chem. 2009, 393, 393-397.

(15) Bouyssiere, B.; Leonhard, P.; Pröfrock, D.; Baco, F.; Garcia, C. L.; Wilbur, S.; Prange, A. J. Anal. At. Spectrom. 2004, 19, 700-702.

(16) Heilmann, J.; Heumann, K. G. Anal. Bioanal. Chem. 2008, 390, 643-653.

(17) Heilmann, J.; Heumann, K. G. Anal. Chem. 2008, 80, 1952 1961.

(18) Bolea-Fernandez, E.; Balcaen, L.; Resano, M.; Vanhaecke, F. J. Anal. At. Spectrom. 2017, 32, 1660-1679.

(19) Vetere, A.; Pröfrock, D.; Schrader, W. Angew. Chemie - Int. Ed. 2017, 56, 10933-10937 
(20) Calderon-Celis, F.; Diez-Fernandez, S.; Costa-Fernández, J. M. Encinar, J. R.; Calvete, J. J.; Sanz-Medel, A. Anal. Chem. 2016, 88 , 9699-9706.

(21) Nelson, J.; Hopfer, H.; Silva, F.; Wilbur, S.; Chen, J.; Shiota Ozawa, K.; Wylie, P. L. J. Agric. Food Chem. 2015, 63, 4478-4483.

(22) Freije-Carrelo, L.; Moldovan, M.; García Alonso, J. I.; Thanh Vo, T. D.; Encinar, J. R. Anal. Chem. 2017, 89, 5719-5724.
(23) Chainet, F.; Lienemann, C. P.; Ponthus, J.; Pécheyran, C.; Castro, J.; Tessier, E.; Donard, O. F. X. Spectrochim. Acta B 2014, 97, 49-56.

(24) Pröfrock, D.; Leonhard, P.; Wilbur, S.; Prange, A. J. Anal. At. Spectrom. 2004, 19, 623-631.

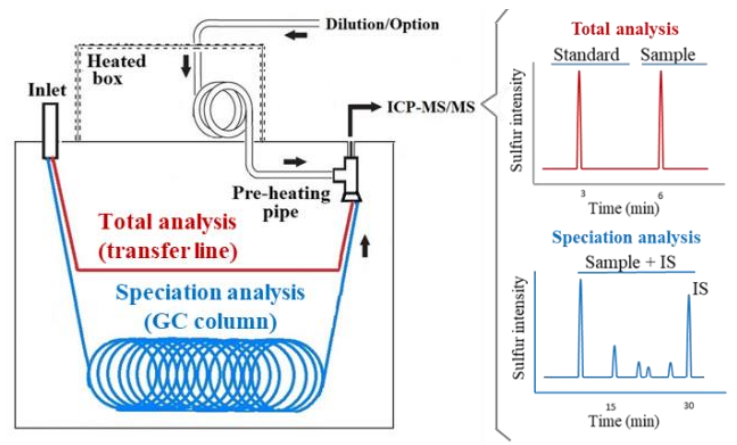

Table of Contents 